\title{
Spontaneous Cervical Spinal Epidural Hematoma
}

Katie Rinne, MBBS; Sunil Gopisetty, MRCEM

\section{A 68-year-old woman presented with sudden-onset right arm and leg weakness, as well as right-sided neck pain.}

U nilateral weakness is a common ED presentation with a diverse etiology, including stroke. ${ }^{1,2}$ Studies have reported a misdiagnosis rate of stroke and transient ischemic attack of approximately $10 \% .^{3}$ This case presents an unusual stroke mimic where treatment with an anticoagulant could have led to adverse outcomes. It also highlights the importance of considering a spontaneous cervical spinal epidural hematoma (SCSEH) as a stroke mimic. This is especially pertinent when a patient's symptoms are not fully consistent with an acute stroke, in order to avoid potentially dangerous anticoagulation and allow for prompt treatment of the hematoma.

\section{Case}

A 68-year-old woman presented to the ED via ambulance with the complaint of stroke-like symptoms. She had a 2-hour history of sudden-onset right arm and leg weakness with loss of sensation, and right-sided neck pain. She also complained of a mild right-sided headache, which had an insidious onset and had been present for the past 2 days. She had recently finished a course of antibiotics for a urinary tract infection. There was no history of trauma. The patient had no significant medical history, was taking no medications, and was a nonsmoker with a normal body mass index. Her family history was significant for cerebral vascular accidents.

On arrival at the ED, the patient had a blood pressure of 179/95 mm Hg; her other vital signs were normal. On examination, she had a right-sided hemiplegia, with a $0 / 5$ power grading observed for motor strength for both her right arm and leg. She reported paresthesia in dermatomes C4 to C5 and L3 to L5. There was extreme tenderness when her right trapezius and upper paraspinal muscles were palpated, but she had no midline cervical spine tenderness and had full, though painful, range of movement of her neck. Her left side was unaffected. She had normal cranial nerves, no higher cortical dysfunction, a Glasgow Coma Scale (GCS) score of 15, and complete control of her bladder and bowel.

A computed tomography angiogram (CTA) of the carotid arteries and a CT scan of the head were ordered to rule out acute stroke and carotid artery dissection. The CT scan of the head showed no acute bleeding or evidence of infarction. However, the CTA raised suspicion of an SCSEH.

Subsequent magnetic resonance imaging showed a right posterior epidural lesion measuring 3 to $3.5 \mathrm{~cm}$ in length and 8 to $9 \mathrm{~mm}$ in maximal thickness, extending from the mid-

Dr Rinne is the foundation doctor in the emergency department at Sunderland Royal Hospital, Sunderland, England. Dr Gopisetty is the specialist registrar in the emergency department at Sunderland Royal Hospital, Sunderland, England. 
point of C2 to the C3-C4 disc level. The lesion compressed the spinal cord, and this stenosis was made worse by posterior disc osteophytes. There was a single strongly enhancing vessel projecting within the hematoma, raising the suspicion of an active bleed (Figure).

Blood tests, including a coagulation screen, were normal and an electrocardiogram showed normal sinus rhythm at 57 beats/minute.

The patient was transferred to the neurosurgery team at the tertiary center, where she underwent an emergency C2-C3 laminectomy that day. She was discharged 6 days later and has made a full recovery with physiotherapy, regaining full function of her right arm and leg.

\section{Discussion}

Spontaneous cervical spinal epidural hematomas are rare, with an estimated annual incidence of 0.1 cases per 100,000 people. ${ }^{4}$ The etiology is largely unknown, but SCSEH has been attributed to a venous source. ${ }^{5}$ Multiple predisposing factors have been reported, including coagulopathies, anticoagulation, disc herniation, vascular malformations, neoplasms, and idiopathic causes. ${ }^{3,4}$

The most common presentation of an SCSEH is sudden onset of interscapular or cervical pain with paresthesia and even paralysis. Paraplegia and quadriplegia are common; however, hemiplegia as a presentation of an SCSEH is rarely reported in the literature. ${ }^{6-8}$

In a case report, Wang et $\mathrm{al}^{6}$ described a similar presentation and management of an SCSEH in the ED as our case. However, the patient in that report was initially treated for a stroke with heparin, and the authors commented that the unnecessary anticoagulation could have caused expansion of the hematoma and subsequent spinal cord compression. ${ }^{6}$

Both our patient and the patient described by Wang et $\mathrm{al}^{6}$ presented with hemiplegia, which is more commonly seen as a presenting feature in stroke and is a rarely reported presentation of an SCSEH. However, neck

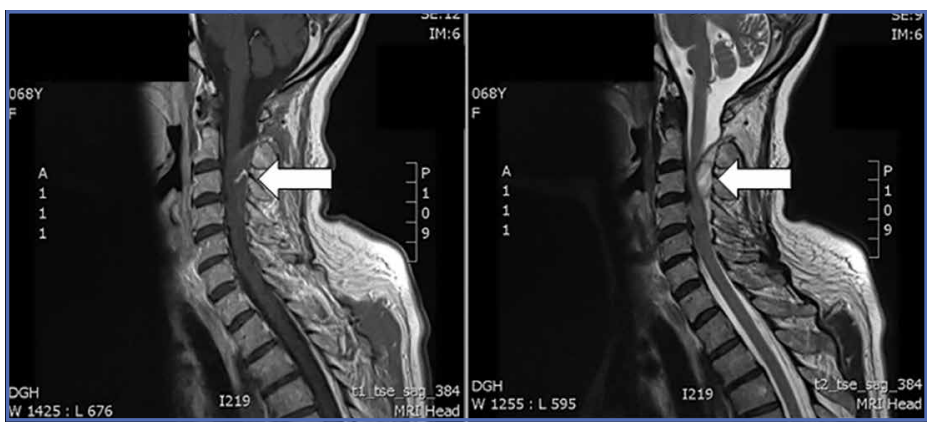

Figure. Magnetic resonance imaging shows a right posterior epidural lesion (arrows) at the C2-C4 level, with an enhancing vessel projecting within the hematoma.

pain is not a classical presenting feature in stroke, and this prompted us to order the CTA of the carotid arteries and CT scan of the head. This ultimately led to the correct diagnosis and prompt management, avoiding unnecessary and potentially dangerous anticoagulation.

\section{Conclusion}

Hemiplegia is an important, though rarely reported, presentation of an SCSEH and should not be misdiagnosed as an acute stroke. Neck pain in a patient presenting with unilateral weakness should be a red flag that prompts the emergency physician (EP) to search for alternative diagnoses to stroke. If a patient with an SCSEH presents to the ED, prompt and accurate recognition by an EP allows for early surgical intervention, which improves clinical outcome, aids neurological recovery, and minimizes long-term sequales. ${ }^{6}$

\section{References}

1. Nickel C, Nemec M, Bingisser R. Weakness as presenting symptom in emergency department. Swiss Med Wkly. 2009;139(17-18):271-272.

2. Asimos AW, Hockberger RS, Grayzel J. Evaluation of the adult with acute weakness in the emergency department. UpToDate web site. Available at: http:// www.uptodate.com/contents/evaluation-of-theadult-with-acute-weakness-in-the-emergency-department. Accessed April 12, 2016.

3. Pope JV, Edlow JA. Avoiding misdiagnosis in patients with neurological emergencies. Emerg Med Int. 2012;2012:949275.

4. Baek B, Hur J, Kwon Ki, Lee HK. Spontaneous spinal epidural hematoma. J Korean Neurosurg Soc. 2008; 44(1):40-42

5. Fukui M, Swarnkar A, Williams R. Acute spontaneous spinal epidural hematomas. AJNR Am J Neuroradiol.1999;20(7):1365-1372.

6. Wang CC, Chang CH, Lin HJ, Lin KC, Kuo JR. Misdiagnosis of spontaneous cervical epidural haemorrhage. Eur Spine J. 2009;18(Suppl 2):210-212.

7. Hsieh CF, Lin HJ, Chen KT, Foo NP, Te AL. Acute spontaneous cervical spinal epidural hematoma with hemiparesis as the initial presentation. Eur J Emerg Med. 2006;13(1):36-38.

8. Dimou J, Jithoo R, Bush S. A patient with delayed traumatic cervical spinal epidural hematoma presenting with hemiparesis. J Clin Neurosci. 2010;17(3): 404-405. 